\author{
Military Technical College \\ Kobry El-Kobbah, \\ Cairo, Egypt.
}

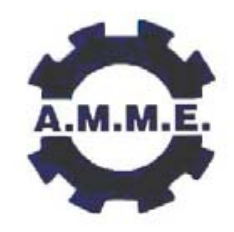

\title{
APPLICATION OF PRESSURE SENSTIVE FOIL TECHNIQUE TO VISULAIZE SUBSONIC JET FLOW OVER A PLATE
}

\author{
$13^{\text {th }}$ International Conference \\ on Applied Mechanics and \\ Mechanical Engineering.
}

\author{
IBRAHIM $^{*}$ M.K., MORI ${ }^{* *}$ K. and NAKAMURA ${ }^{* * *} Y$.
}

\begin{abstract}
The present study proposes new technique to implement pressure sensitive coating on porous anodized aluminum. The new technique is called pressure sensitive foil (PSFoil). It is based on the conventional anodized aluminum pressure sensitive paint (AAPSP) technique. In this technique, a very thin aluminum foil is coated with pressure sensitive paint using anodization method. The resulting pressure sensitive foil (PS-Foil) can be stick over any flat surface using a very thin silicon layer. The PS-Foil technique shows very fast time response as conventional porous anodized aluminum and high spatial resolution compared with conventional PSP binder based techniques. It can also be applied to any existing model surface without the need to fabricate the model from aluminum. The total thickness of the aluminum foil and silicon layer is as small as 250 micro-meters. The paint employed in the present research is Bathophen Ruthenium Chloride $\left(\mathrm{Ru}\left(\mathrm{ph}_{2}\right.\right.$-phen) or $\left.\mathrm{Ru}(\mathrm{dpp})\right)$. Commercially available inexpensive, high quality, 14-bit, CCD camera is used to capture the PSP images. Intensity based method and "in-situ" calibration procedure are used to obtain the calibrated PSP images. The proposed technique was applied to measure the pressure distribution over a flat plate due to subsonic jet impingement at different impingement angles. The resulting PSP images showed high spatial resolution of the pressure distribution and pressure difference as low as $0.5 \mathrm{kPa}$ can be measured.
\end{abstract}

\section{KEY WORDS}

Pressure Sensitive Paints (PSP), Pressure Sensitive Foil Technique, Subsonic Jet, JetPlate Impingement, Bathophen Ruthenium Chloride, and in-situ Calibration Technique

\footnotetext{
Lecturer, Aerospace Engineering Department, Faculty of Engineering, University of Cairo, Giza, 12613 Egypt, email: mkhalil@eng.cu.edu.eg. Currently Assistant Professor, Aerospace Engineering Department, Graduate School of Engineering, Nagoya University, Furo-cho, Chikusa-ku, Nagoya 464-8603, Japan. email: khalii@nuae.nagoya-u.ac.jp.

** Lecturer, Aerospace Engineering Department, Graduate School of Engineering, Nagoya University, Furo-cho, Chikusa-ku, Nagoya 464-8603, Japan. email: mori@nuae.nagoya-u.ac.jp.

*** Professor, Aerospace Engineering Department, Graduate School of Engineering, Nagoya University, Furo-cho, Chikusa-ku, Nagoya 464-8603, Japan. email: nakamura@nuae.nagoyau.ac.jp.
} 


\section{NOMENCLATURE}

$\begin{array}{ll}A & : \text { Stern-Volmer relation first constant } \\ B & : \text { Stern-Volmer relation second constant } \\ D & : \text { Nozzle exit diameter } \\ I & : \text { Luminescent intensity } \\ I_{\text {ref }} & : \text { Luminescent intensity at a reference condition } \\ M & : \text { Mach number } \\ P & : \text { Total pressure } \\ p & : \text { pressure } \\ T & : \text { Temperature } \\ x, y, z & : \text { Cartesian coordinates } \\ R u\left(p h h_{2}-p h e n\right) & : \text { Bathophen Ruthenium Chloride } \\ A A & : \text { Anodized aluminum } \\ C C D & : \text { Charge-Coupled Device } \\ I C C D & : \text { Intensified Charge-Coupled Device } \\ P M T & : \text { Photomultiplier Tubes } \\ P S P & : \text { Pressure Sensitive Paints } \\ T S P & : \text { Temperature Sensitive Paints } \\ \gamma & : \text { Specific heat ratio } \\ \lambda & : \text { Luminescent emission wave length } \\ \tau & : \text { Response time } \\ \theta & : \text { Plate inclination angle in degrees } \\ \text { Subscripts } & : \text { Nozzle exit condition } \\ \text { exit } & : \text { Maximum value } \\ \text { max } & : \text { Minimum value } \\ \text { min } & : \text { reference condition } \\ r e f & : \text { Cartesian coordinates } \\ x, y, z & \text { Ambient condition } \\ \infty & \end{array}$

\section{INTRODUCTION}

Pressure sensitive paint is a powerful tool for measuring the pressure distribution of wind tunnels models. The PSP is a luminescent coating consisting of a luminophore and a binder. The luminescent coating emitting radiation under excitation by an incident light. The luminescent emission from the coating can be affected by certain physical processes. The main photophysical process in PSP is oxygen quenching that cause a decrease of the luminescent intensity as the partial pressure of oxygen or air pressure increases. Therefore, the PSP binder must be oxygen permeable.

There are two basic PSP implementation techniques; the first is a conventional polymer PSP and the other is porous PSP technique. Figures 1(a) and 1(b) shows their schematic comparison. In the conventional polymer PSP as shown in Fig. 1(a), oxygen molecules in the working gas permeate into a polymer layer binder and quench the luminescence. The main advantage of using a polymer as a binder for PSP is its simplicity in application. However, its time response is slow compared with porous PSP technique. 
In contrast, as illustrated in Fig. 1(b), a porous PSP has a much larger open surface to which luminophore molecules are directly applied; oxygen molecules can directly quench the luminescence without having to permeate into a binder layer. Therefore, the use of a porous material as a binder for PSP offers two advantages. First, it can achieve a very fast time response (in the order of microseconds) for unsteady PSP measurements; secondly, it makes possible PSP measurement at cryogenic temperature at which oxygen diffusion is prevented through a conventional homogeneous polymer [1]. This technique has two main disadvantages. The first, it has a high temperature and moisture dependency; secondly, the implementation of the techniques not easy.

Kurita et al., [2] proposed a method that utilized non-oxygen permeation binder to support the luminophore as shown in Fig. 1(c). The technique called semi-binder PSP. The technique showed good results at hypersonic speeds and supersonic speeds. However, it was reported also that it has poor mechanical property in which the luminophore concentration over the model surface decreases with time due the flow field interaction with the surface supported luminophore.

PSP measurement is challenging in unsteady and low speed flows where a change in air pressure is very small. Unsteady application required very fast time response of PSP and minimization of temperature effect. High gas diffusive material are needed as a binder for the unsteady PSP, because the time response of PSP depends on gas diffusivity in the binder. Until now, three materials have been available as binder: 1) commercial porous silica thin-layer chromatography (TLC-PSP), 2) porous anodized aluminum (AA-PSP) and 3) polymer with hard ceramic particles (PC-PSP). The TLCPSP and AA-PSP exhibit a quite short response time of the order of $10 \mu \mathrm{sec}$ [3].

In low-speed applications the major error sources include but not limited to, the temperature effect, image misalignment, and CCD camera noise. These noise sources must be minimized to obtain acceptable quantitative pressure results at low speeds [1]. Those sources of errors suggest pushing PSP instrumentation to the limit and using pressure correction methods for other sources like temperature and image misalignment.

In the present study, unsteady and low speed flow applications are considered. New implementation of the well knows AA-PSP technique is proposed in this paper. The proposed technique is called pressure sensitive foil (PS-Foil). In this technique, a very thin aluminum foil is coated with pressure sensitive paint using anodization method. The resulting PS-Foil can be stick over any flat surface using a very thin layer of silicon. Recently, AA-PSP showed very promising unsteady characteristics [3-5]. The proposed technique has two advantages. First, it can be applied on any existing model without being manufacturing from aluminum; secondly, it is very suitable for models that cannot be manufactured from aluminum due to stress consideration like high speed rotating blades. However, this technique works well for flat surface model or model with high radius of curvature; it is difficult to apply on complex models. In this paper both unsteady characteristics of the PS-foil and steady low-speed performance will be investigated. The steady low-speed test case chosen here is the subsonic jet-flat plate interaction. The flow field associated with a subsonic or sonic jet impinging on a flat plate has been studied extensively [6]. The jet-flat plate impingement exists in a wide range of applications. In the previous studies, the spatial resolution of pressure taps 
required for a complete survey of the impingement surface is not practical. By applying the PSP technology to the obliquely impinging jet high resolution pressure distributions on the impingement surface can be acquired and better understanding of the aerodynamic interaction can be achieved.

\section{EXPERIMENTAL SETUP}

\section{Test Facility}

Experiments were conducted in the open jet facility at Department of Aerospace Engineering, Nagoya University. A subsonic jet is exhausted inside an anechoic chamber as shown in Fig. 2(b). The nozzle employed in the present study to produce the jet flow is a convergent nozzle with an exit diameter of $10 \mathrm{~mm}$, which is attached to a cylindrical plenum chamber that has a diameter of $220 \mathrm{~mm}$ and a length of $400 \mathrm{~mm}$. The plate, which causes interaction with the jet, is made of aluminum alloy containing $\mathrm{Mg}, \mathrm{Fe}, \mathrm{Cr}$ and Si (Japanese Industrial Standards (JIS) A5052) and has a length of 300 $\mathrm{mm}$, a width of $200 \mathrm{~mm}$, and a thickness of $10 \mathrm{~mm}$. Figure 2(a) shows schematic of the experimental setup employed in the present study. The plate is supported over traverse system that has four degree of freedoms. It can move the plate in $x, y$, and $z$ directions with respect to the center of nozzle exit. The plate inclination angle can be varied from $\theta=0^{\circ}$ to $\theta=30^{\circ}$. The coordinate system employed in the present study is depicted in Fig. 2(c).

High-pressure air is supplied from a tank with a volume of $12 \mathrm{~m}^{3}$, where air is stored at a pressure of $12 \mathrm{kgf} / \mathrm{cm}^{2}$. This tank is connected to the plenum chamber via a high pressure pipe with an inner diameter of one inch. A high precision pressure regulator and a solenoid valve were used in order to control the pressure inside the plenum chamber to within an accuracy of $0.25 \%$. The solenoid valve is opened or closed via a signal that comes from the data acquisition PC (DAQ-PC) with a data acquisition board (National Instruments, PCl-6035E) The DAQ-PC is connected to a 6 channel DC strain amplifier (KYOWA DPM-6H) with a frequency response of $5 \mathrm{kHz}$, which is in turn connected to various pressure transducers and load cells. The pressure transducers are used to monitor the pressure inside the plenum chamber as well as measuring the pressure on plate surface. Twenty pressure taps were used to measure the pressure on the plate centerline, where the jet impinges the plate, using a single pressure transducer and scanivalve for in-situ PSP calibration procedures.

To explore the time response of the proposed PS-Foil, a simple shock tube experiments was conducted. Fig. 3(a) shows the schematic of the simple shock tube experiment. The total length of the driven section of the shock tube is 2 meters. The test specimen is placed at one side inside the driven section of the tube at a distance of 1.4 meter from the diaphragm. On the opposite side of the test specimen optical window was installed to provide optical access to the test specimen. The excitation light source is 8 blue LEDs (NICHIA NSPB510S, wavelength: 450-480 $\mathrm{nm}$ ). The detector is a photomultiplier tube (Hamamatsu R374). A long pass filter, which can pass luminescence with a wave-length longer than $\lambda=580 \mathrm{~nm}$ at $80 \%$ intensity, is installed in front of the photomultiplier tube. At the same location of specimen, a flush mount high speed pressure transducer (Kulite XCS-062-15D) is installed. This transducer is connected to a single channel dynamic DC strain amplifier (SAN-EI 6M71) with a 
frequency response of $100 \mathrm{kHz}$. Both the photomultiplier tube and the pressure transducer output signals are recorded using DAQ-PC.

In data acquisition, transducer zero errors were monitored before each run, and the plenum chamber and ambient pressures were also checked for each run, which were used to normalize measured data.

\section{Pressure Sensitive Foil Preparation}

The preparation of the pressure sensitive foil using AA-PSP is described briefly. Detailed information about AA-PSP is found in literature $[3,4,5]$.

\section{Materials:}

Aluminum foil of thickness $200 \mu \mathrm{m}$ containing Mg, Fe, $\mathrm{Cr}$ and Si (Japanese Industrial Standards (JIS) 1050) was employed as the material of the foil. The luminophore was Bathophen Ruthenium Chloride (Ru(ph - -phen) or $R u(d p p))$.

2. Pretreatment:

An aluminum sheet was dipped in a $2 \%$ sodium hydroxide solution for 2 minutes to remove an excess oxidized layer. The surface was rinsed by water after this process

3. Anodization:

A constant current density $17.5 \mathrm{~mA} / \mathrm{cm}^{2}$ was applied to the aluminum sheet, which was connected to anode in 1 molar sulfuric acid at $10^{\circ} \mathrm{C}$.

4. Post-Anodization:

The anodized sheet was dipped in a $5 \%$ phosphoric acid for 20 minutes at $30{ }^{\circ} \mathrm{C}$. After this process, the sheet was rinsed by water and then dried

5. Dipping:

The luminophores molecules are adsorbed on the anodized aluminum. The aluminum model was dipped in the dye solution, The dye solution was Bathophen Ruthenium Chloride (Ru(ph $\mathrm{ph}_{2}$-phen) or $\mathrm{Ru}(\mathrm{dpp})$ ) dissolved in dichloromethane $\left(\mathrm{CH}_{2} \mathrm{Cl}_{2}\right)$ with ratio of $28 \mathrm{mg}$ of Bathophen Ruthenium Chloride (Ru(ph -phen) or $\mathrm{Ru}(\mathrm{dpp})$ )to $40 \mathrm{ml}$ dichloromethane $\left(\mathrm{CH}_{2} \mathrm{Cl}_{2}\right) .20$ seconds dipping was conducted at $298 \mathrm{~K}$.

6. Binding:

The foil then was sticking to the flat plate using a $50 \mu \mathrm{m}$ silicon layer.

\section{PSP Hardware and Software}

The photo-detector employed in the present study is a CCD camera, 14 bit Canon EOS D40 with resolution of $2592 \times 3888$ pixels. An array of 100 power blue LEDs (NICHIA NSB083T, wavelength: $450 \mathrm{~nm}$ ) was employed as the excitation light. The image capture rate was one frame per $1 / 4 \mathrm{sec}$. Experiments show that the capture rate is highly dependent on the excitation light and shutter opening. The camera is completely controlled through PC via serial port using Canon Remote Capture software. In all experiments the luminescence that CCD camera can detect was filtered to prevent unnecessary illuminating luminescence. The filter is a long pass filter, which can pass luminescence with a wave-length longer than $\lambda=580 \mathrm{~nm}$ at $80 \%$ intensity.

To obtain the calibrated PSP images, intensity based method was employed in the present study. The basic processing procedure in the intensity-based method of PSP is 
by calculating the ratio between the wind-on image and the wind-off reference image to correct the effects of non-homogenous illumination, uneven paint thicknesses and nonuniform luminophore concentrations. However, this proportioning procedure is complicated by model deformation induced by aerodynamic loads, which results in misalignment between the wind-on and wind-off images. By increasing the plate thickness and stiffening the support system one can reduce this deformation, nevertheless it can not be completely removed and produces noise in the resulting image. Therefore additional correction procedures are required to eliminate the error sources associated with model deformation. These correction procedures are not implemented in the present study. The temperature effect of PSP and self-illumination noise are not implemented here as well. However, to reduce the temperature change between wind-off and wind-on images, wind-off images was taken immediately after the wind-on image not before. Noise caused by darkness is reduced in the current work by subtraction dark image from the data image. A code is developed for post-processing and calibration. Simultaneous pressure measurements and image capturing processing capability is included in this post-processing code for online processing of PSP images.

\section{RESULTS AND DISCUSSION}

The subsonic jet considered in the present study had Mach number of 0.6. Five plate inclination angle, $\theta$ were considered at the present study, specifically, $\theta=0^{\circ}, 5^{\circ}, 10^{\circ}$, $15^{\circ}$ and $20^{\circ}$. The unsteady characteristics and flow field results will be discussed in the following.

\section{Unsteady Characteristics}

The unsteady characteristics of the proposed PS-Foil technique was explored using the simple shock tube experiments described in section test facility. Figure 3(b) shows a typical pressure signal from PS-Foil, semi-binder PSP, pressure transducer along with the theoretical pressure jumps associated with the incident and reflected normal shock waves. The PS-Foil was able to follow the sharp pressure rises after the incident and reflected shock waves passed through the PSP test specimen exited by the blue LED. Figure 3(c) shows the normalized pressure signal from PS-Foil, semi-binder PSP and pressure transducer. It was found that the normalized pressure response to step input is well represented by first order model described by the following equation:

$$
\frac{p-p_{\min }}{p_{\max }-p_{\min }}=1-e^{\frac{t}{\tau}}
$$

where $\tau$ is the response time. $\tau$ was found to be 20,30 , and $500 \mu$ sec for pressure transducer, PS-Foil, and semi-binder PSP, respectively. The present value for response time of PS-Foil is the same as that of AA-PSP of thickness $4.3 \mu \mathrm{m}$ measured by Sakaue [7].

\section{Jet Flow Visualization}

In oxygen quenching process mentioned in the introduction, the luminescent emission of a molecule, when exposed to oxygen, can be modeled in many cases by the SternVolmer relation [8]. 


$$
\frac{I_{r e f}}{I}=A(T)+B(T) \frac{p}{p_{r e f}}
$$

where $I_{\text {ref }} / I$ are luminescent intensity and air pressure at a reference condition, respectively. The Stern-Volmer coefficients $A$ and $B$, which are temperature-dependent due to the thermal quenching [1], are experimentally determined by calibration. Thermal quenching is the mechanism in temperature sensitive paints (TSP) that reduces the luminescent intensity as temperature increases. Theoretically speaking, the intensity $I_{\text {ref }} / l$ can eliminate the effects of non-uniform illumination, uneven coating and non homogenous luminophore concentration in PSP. In typical tests in a wind tunnel, $I_{\text {ref }}$ is taken when the tunnel is turned off and hence it is often called wind-off image or intensity; likewise, $I$ is called the wind-on image or intensity.

The acquired images can be calibrated using in-situ calibration procedure [1] which can be described briefly as follow: $I_{\text {ref }} l$ is called the un-calibrated PSP image. From this image, PSP data or intensity is collected and averaged around the pressure taps points (that are used for pressure measurement). Fig. 4 shows a curve fit of the $I_{\text {ref }} / l$ as a function of $p / p_{\text {ref }}$ at the pressure tap locations for plate inclination angle $\theta=0^{\circ}$. The general trend is shown as a solid line while the circles are the pressure tap data. From this linear equation of the trend line, the Stern-Volmer coefficients $A$ and $B$ can be calculated. Table 1 summaries the Stern-Volmer coefficients $A$ and $B$ computed for varies plate inclination angles. High value of $B$ coefficient indicates good agreement between pressure tap data and PSP data.

Table 1: Computed Stern-Volmer coefficients from in-situ calibration procedures

\begin{tabular}{lll}
\hline \hline$\theta$ & $A$ & $B$ \\
\hline $0^{\circ}$ & -11.3 & 12.3 \\
$5^{\circ}$ & -4.79 & 5.75 \\
$10^{\circ}$ & -4.28 & 5.27 \\
$15^{\circ}$ & -2.69 & 3.69 \\
$20^{\circ}$ & -2.31 & 3.31 \\
\hline \hline
\end{tabular}

Figure 5 shows the pressure distribution along the plate centerline at $y=0$ for both pressure taps data and the extracted pressure data from calibrated PSP images at different plate inclination angle. At low inclination angle, specifically $\theta=0^{\circ}$ and $5^{\circ}$, there is good agreement between both pressure taps data and PSP data. As the inclination angle increases, specifically $\theta=10^{\circ}, 15^{\circ}$ and $20^{\circ}$, there is large deviation near the nozzle exit and there is a good agreement in the downstream. The deviation occurs in high pressure regions. This is attributed to the temperature effect on PSP data and it is highlight the importance to correct the present PSP data to include the temperature effect. Figure 6 shows pressure images mapped onto the plate surface for different plate inclination angle. The jet signature on the whole plate is clearly captured using the proposed PS-Foil technique. 


\section{CONCLUSION}

New implementation technique for the AA-PSP is presented in this paper. In this technique, a very thin Aluminum foil is coated with pressure sensitive paint using anodization method. The resulting pressure sensitive foil (PS-Foil) can be stick over any flat surface using very thin silicon. Both unsteady characteristics of the PS-foil and steady low-speed performance were investigated. The steady low-speed test case was the subsonic jet of Mach number 0.6 impinging a flat plate at different plate inclination angle. Results showed that the PS-Foil technique has very fast time response $(\tau=30$ $\mu s e c)$ as the very thin conventional porous anodized aluminum and high spatial resolution compared with conventional PSP binder based techniques. The PS-Foil can detect pressure as low as $0.5 \mathrm{kPa}$. However, at high pressure regions, discrepancy between PSP data and pressure tap data is observed. This discrepancy might be attributed to the temperature effect which was not included in the present study.

\section{REFERENCES}

[1] T. Liu and J. P. Sullivan. Pressure and Temperature Sensitive Paints. pringer, 2005.

[2] Mitsuru Kurita, Takumi Okada, Ryo Sakuma, and Yoshiyaki Nakamura. Pressure and Temperature Sensitive Paint Measurement in Shock Tunnel. Journal of the Visualization Society of Japan, 22(1), 2002.

[3] M. Kameda, T. Tabei, K. Nakakita, H Sakaue, and K. Asai. Image Measurement of Unsteady Pressure Fluctuation by a Pressure-Sensitive Coating on Porous Anodized Aluminum. Measurement Science and Technology, 16:2517-2524, 2005.

[4] Hirotaka Sakaue, Takatoshi Tabei, and Masaharu Kameda. Hydrophobic Monolayer Coating on Anodized Aluminum Pressure Sensitive Paint. Sensors and Actuators B, 119:504-511, 2006.

[5] Hirotake Sakaue. Luminophore Application Method of Anodized Aluminum Pressure Sensitive Paint as a Fast Responding Global Pressure Sensor. Review of Scientific Instruments, 76:1-6, 2005.

[6] Crafton J., Lachendro N., Guille M., Sullivan J. P., and Jordan J. Application of Temperature and Pressure Sensitive Paint to an Obliquely Impinging jet. AIAA Paper, (99-0387), 1999.

[7] Hirotake Sakaue. Porous Pressure Sensitive Paints for Aerodynamic Applications. Master Thesis, School of Aeronautics and Astronautics, Purdue University, West Lafayette, IN, 1999.

[8] M. J. Morries, J. F. Danovan, Kegelman, Schwab J. T., Levy S. D., R. L., and R. C. Crites. Aerodynamic Application of Pressure Sensitive Paint. AIAA Journal, 31:419-425, 1993. 


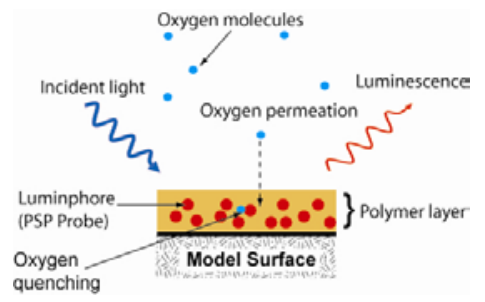

(a) Polymer PSP.

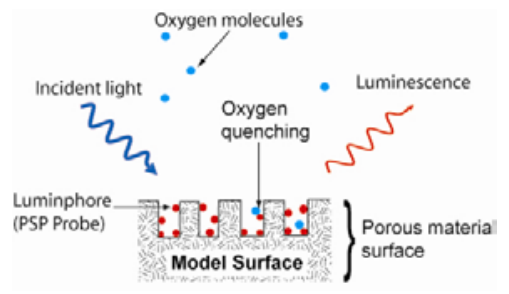

(b) Porous PSP.

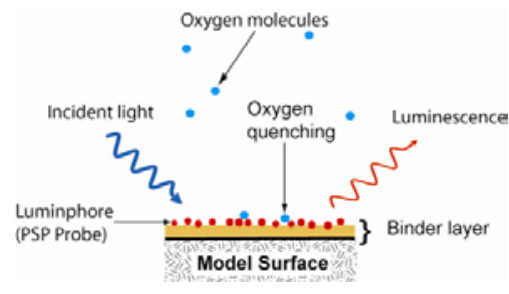

(c) Semi-binder PSP.

Figure 1: Schematic of luminescent painting methods on a surface.

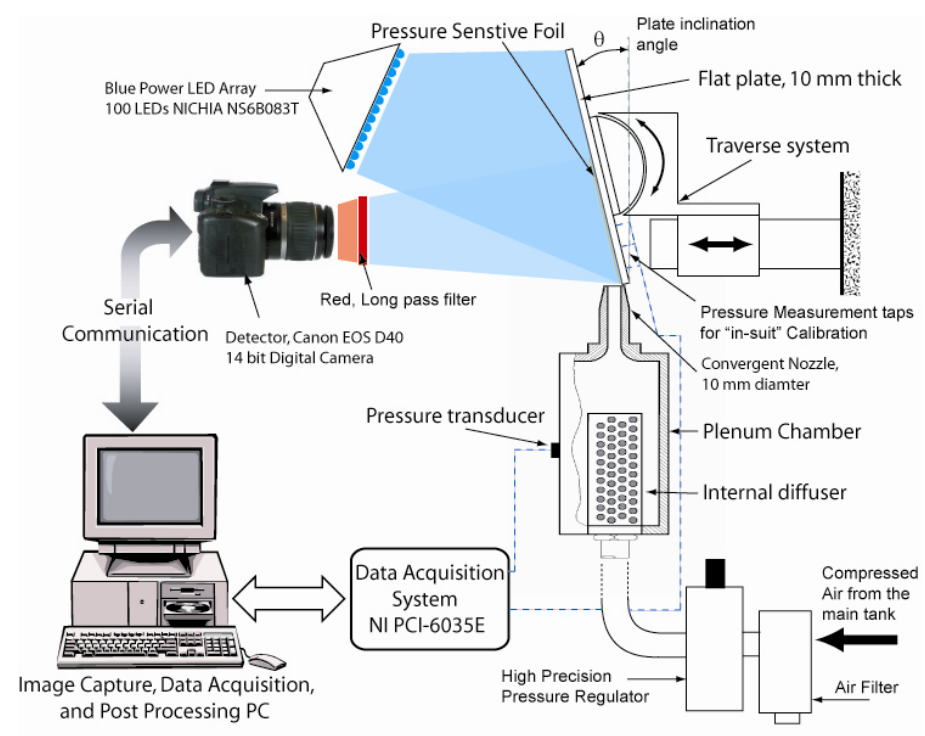

(a) Experimental setup.

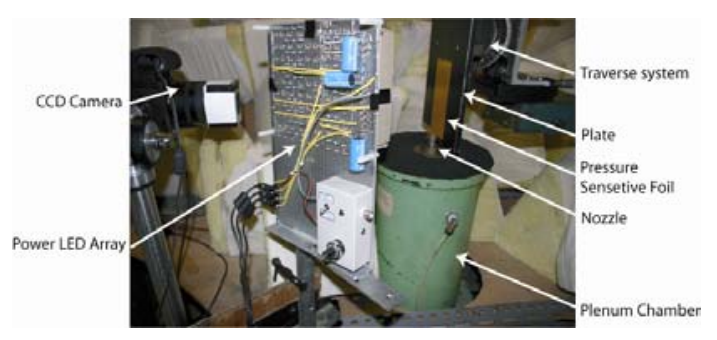

(b) Photo of the experimental setup.

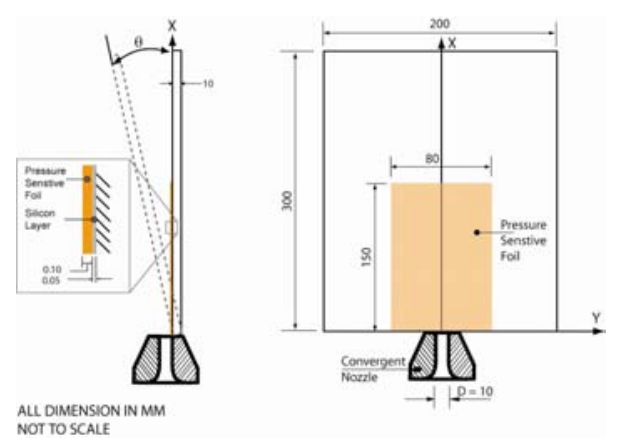

(c) Coordinate system.

Figure 2: Schematic and photo of experimental setup at Aerospace Engineering Department, Nagoya university. 


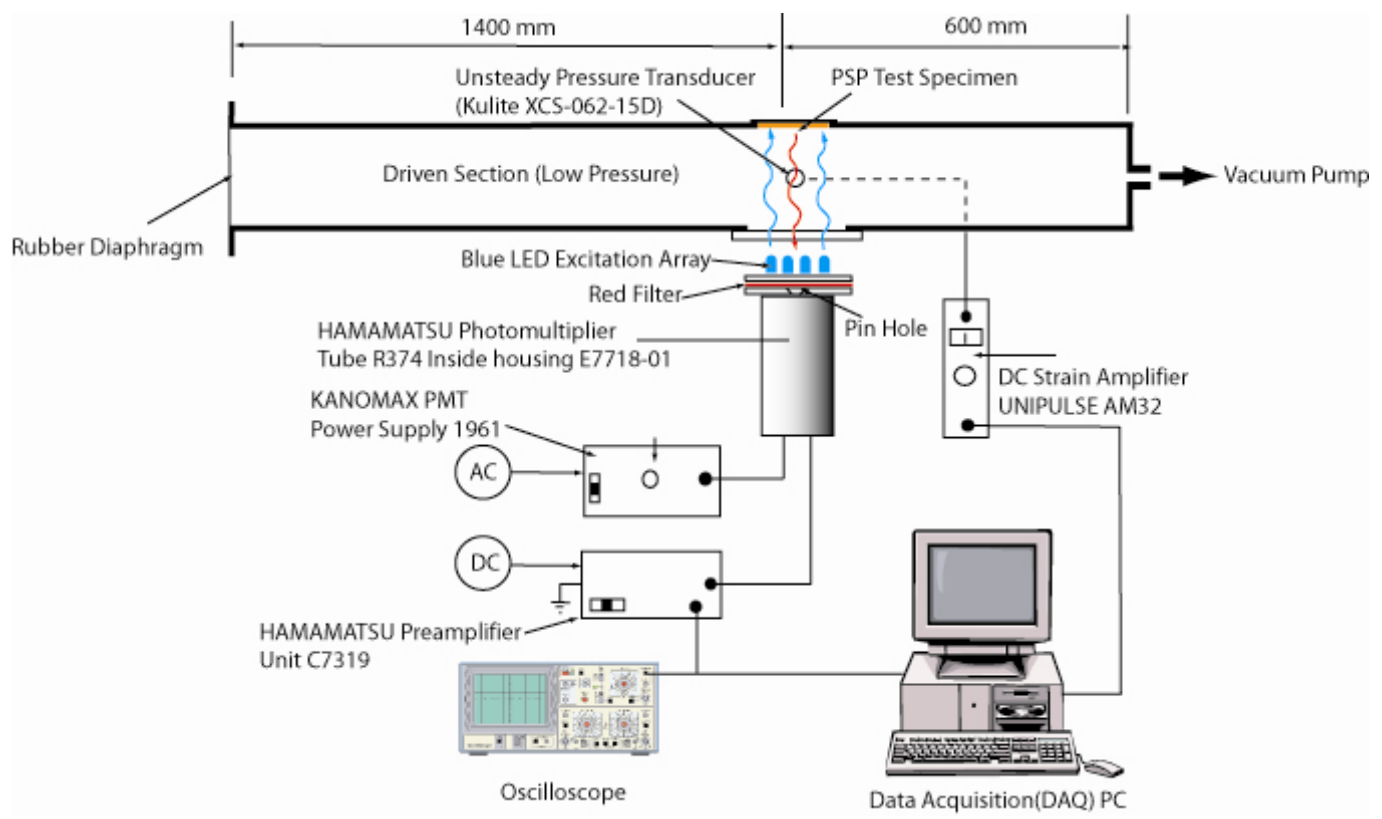

(a) Shock tube experimental setup.

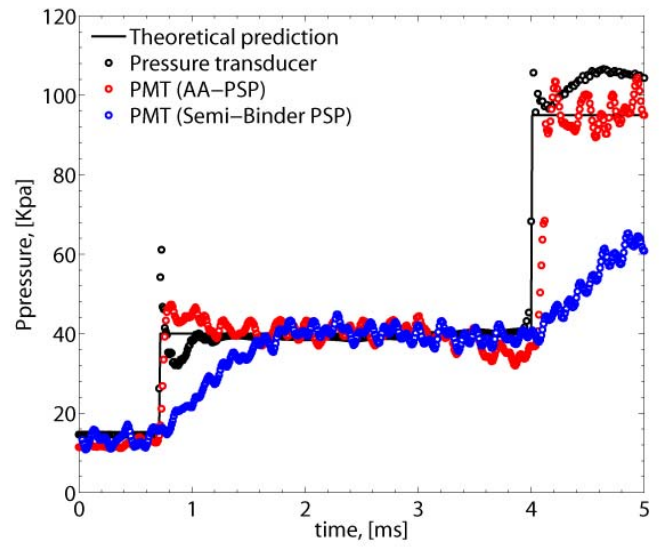

(b) Pressure history.

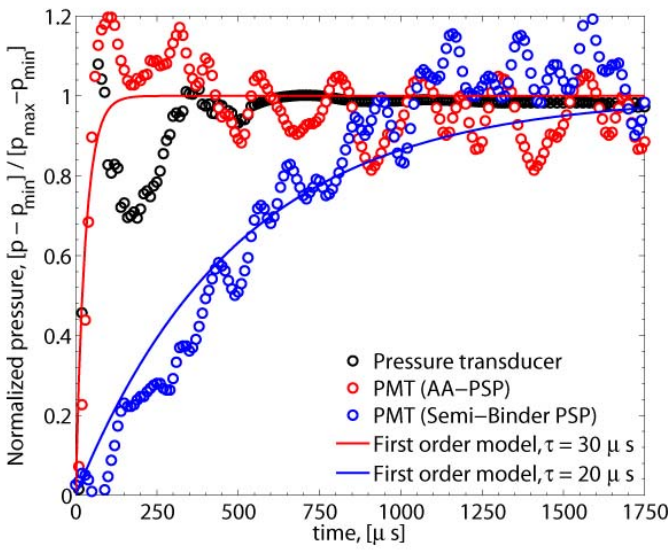

(c) Normalized pressure response.

Figure 3: Pressure history and normalized pressure response of PS-Foil, semi-binder PSP and pressure transducer compared with theoretical calculation of simple shock tube experiment.

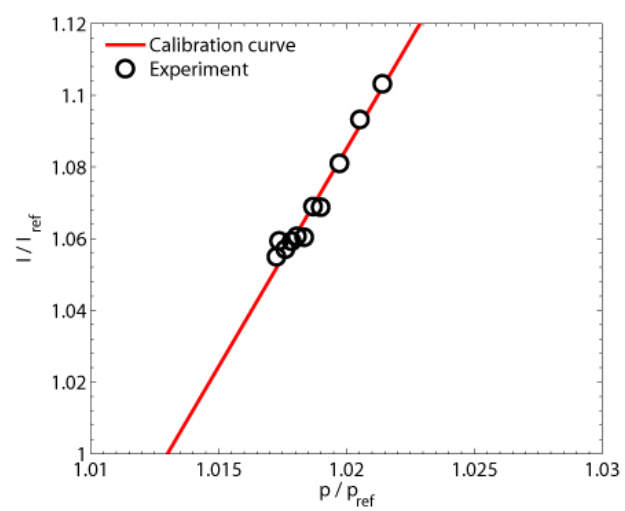

Figure 4: In-situ calibration Stern-Volmer plot for the case of $\theta=0^{\circ}$. 

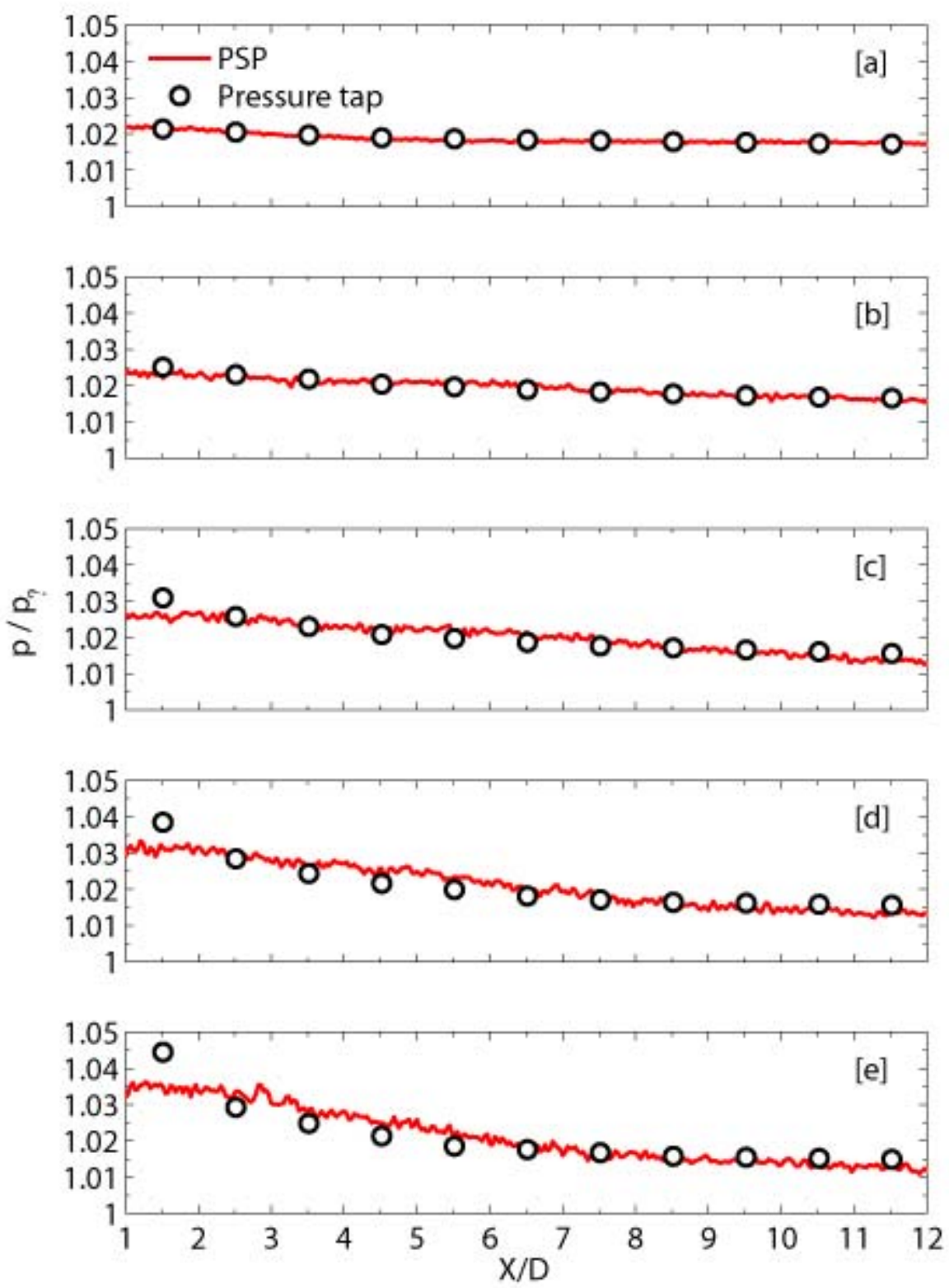

Figure 5: Comparison of PSP and pressure tap data at plate centerline for Mexit $=0.6$ : a) $\theta=0^{\circ}$, b) $\theta=5^{\circ}$, c) $\theta=10^{\circ}$, d) $\theta=15^{\circ}$ and e) $\theta=20^{\circ}$. 


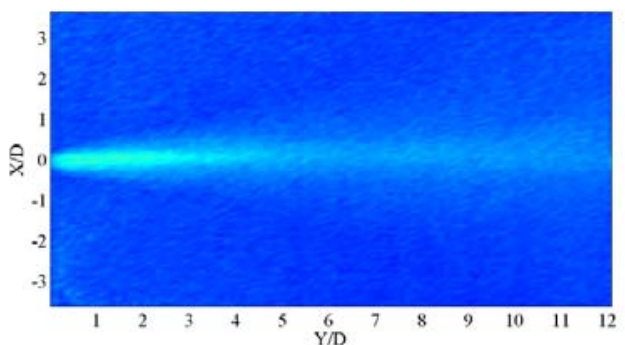

(a) $\theta=0^{\circ}$.

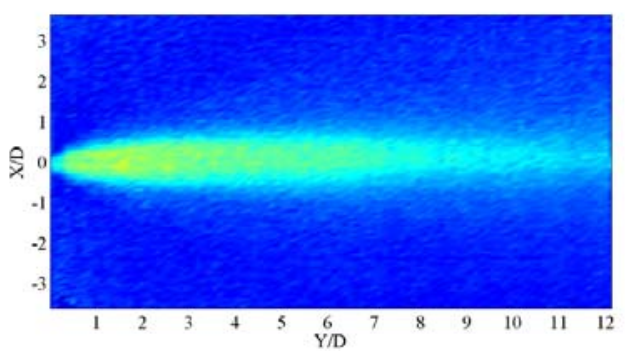

(b) $\theta=5^{\circ}$.

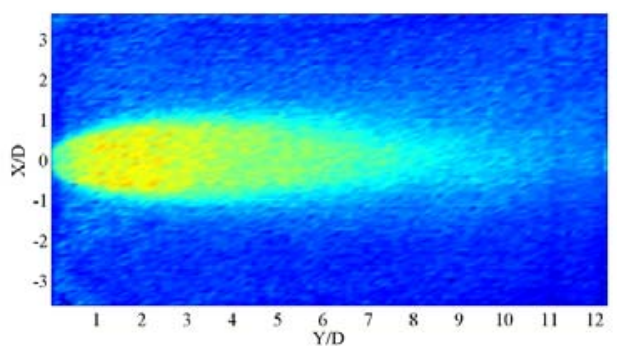

(c) $\theta=10^{\circ}$.

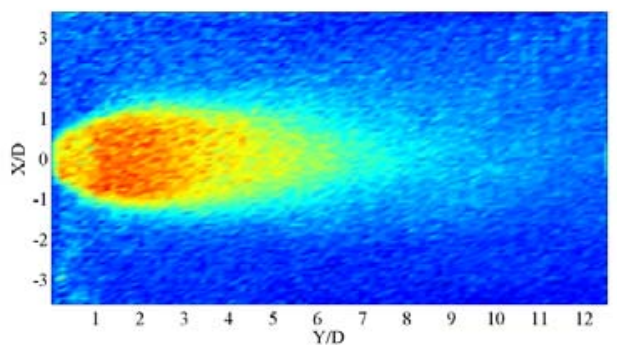

(d) $\theta=15^{\circ}$.

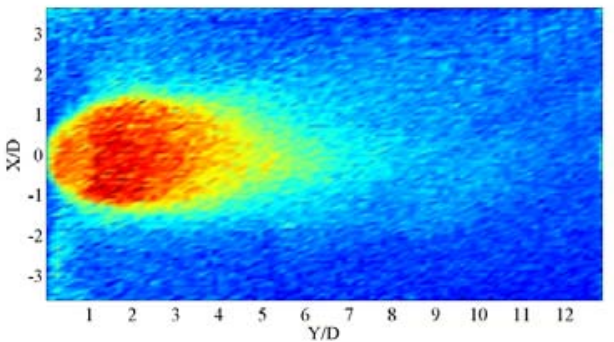

(e) $\theta=20^{\circ}$.

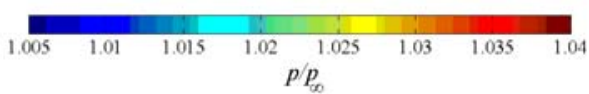

Figure 6: Pressure images mapped onto the plate surface at different plate inclination angle for $M_{\text {exit }}=0.6$. 